\title{
OFET Characteristics of Stretched Poly(3-hexylthiophene) Films
}

\author{
Yoshihito KunUgi, ${ }^{\mathrm{a}, *}$ Yuki Yamada, ${ }^{\text {a }}$ Hiroaki Horiuchi, ${ }^{\mathrm{b}}$ \\ Hiroshi Hiratsuka, ${ }^{\mathrm{b}}$ and Joji OHSHita ${ }^{\mathrm{c}}$
}

\author{
aDepartment of Applied Chemistry, Faculty of Engineering, Tokai University (4-4-1 Kitakaname, Hiratsuka 259- \\ 1292, Japan) \\ ${ }^{b}$ Department of Chemistry and Chemical Biology, Graduate School of Engineering, Gunma University (Kiryu 376- \\ 8515, Japan) \\ 'Department of Applied Chemistry, Graduate School of Engineering, Hiroshima University (Higashi-Hiroshima 739- \\ 8527, Japan)
}

Received September 24, 2009 ; Accepted November 16, 2009

\begin{abstract}
We investigated the field-effect characteristics of mechanically stretched regio-regular poly(3-hexylthiophene) (P3HT) on a supporting poly(vinyl alcohol) (PVA) film. After stretching the P3HT/PVA film, large dichroism was observed in the polarized UV-Vis absorption spectra. The stretched P3HT films were applied parallel or orthogonal to the conduction channel of the transistor. The field-effect mobility was highly anisotropic, with larger mobility along the stretched direction of the films. The mobility increased with increasing stretch ratio $\left(S_{\mathrm{r}}\right)$ for the parallel devices but decreased for the orthogonal devices.
\end{abstract}

Key Words : Polythiophene, Stretching, OFET, Molecular Orientation

\section{Introduction}

Organic field-effect transistors (OFETs) are promising candidates for use in flexible, large-area and low-cost electronic devices, and they have undergone extensive improvements in recent years. Solution-based processes, such as spin-coating, casting, inkjet printing and screen printing, have attracted considerable attention as highly efficient methods for fabrication of OFET devices. ${ }^{1,2)}$ Great effort has been devoted to the development of solution-processable transistor materials and the process techniques for solution-processed OFETs. Recently, a variety of newly developed soluble organic semiconductors have been reported as high performance solutionprocessable transistor materials. ${ }^{3-5)}$ In many cases, the high performance of solution-processed OFETs is due to their well-ordered molecular arrays in their active layers. The hydrophobic intermolecular interaction between alkyl chains leads to enhanced molecular orbital overlap between neighboring molecules, which can enhance carrier mobility in the OFET devices. Molecular ordering in the thin film transistors is also achieved by physical methods such as rubbing and photoalignment method.6.8) Mechanical stretching method (also referred to as the hot drawing method) is also one of the most fundamental and promising techniques for processing uniaxially oriented polymer films. ${ }^{9-11)}$ However, there are very few reports about OFET devices using stretching method.

In this paper, we investigate the OFET characteristics of the mechanically stretched regio-regular poly(3hexylthiophene) (P3HT) polymer films. We select P3HT as a test polymer because it is one of the most popular and well defined organic semiconductors used for solution-processable organic transistors. ${ }^{11-13)}$

\section{Experimental}

P3HT $\left(\mathrm{M}_{\mathrm{w}}=87,000\right)$ with a head-to-tail content of $>97 \%$ was purchased from Aldrich Chemical Co. and used as received. The PVA film (film thickness $=70 \mu \mathrm{m}$ ) was donated by Polatechno Co., Ltd. and cleaned by sonication in ethanol. Chloroform (Aldrich Chemical Co.) was purified by using an $\mathrm{Al}_{2} \mathrm{O}_{3}$ short column just before use.

To determine the stretch ratio $\left(S_{\mathrm{r}}\right)$, we marked the back side of a PVA substrate in intervals of approximately $1 \mathrm{~cm}$. A thin film of P3HT was deposited on the supporting PVA substrate by spin coating a $0.4 \mathrm{wt} \%$ solution of P3HT in chloroform at $2000 \mathrm{rpm}$ for $30 \mathrm{sec}$. This sample film was kept at $65{ }^{\circ} \mathrm{C}$ for 30 min and then mechanically stretched at the stretching speed of $9 \mathrm{~cm}$

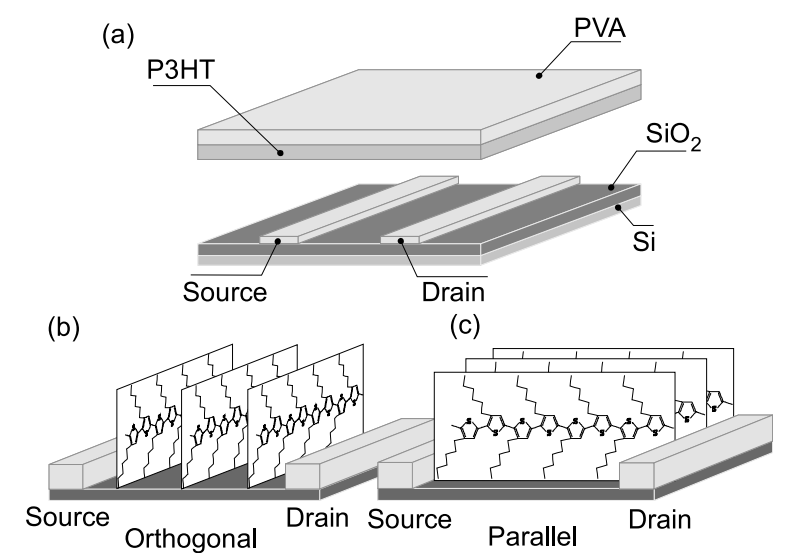

Fig. 1 Schematic illustrations of (a) the bottom-contact type OFET device based on the stretched P3HT film applied (b) orthogonally and (c) parallel to the electric field. 
$\min ^{-1} . S_{\mathrm{r}}$ was defined as $S_{\mathrm{r}}=r_{/ /} / r_{0}$, where $r_{/ /}$and $r_{0}$ are distances between the marks to the stretched direction after and before stretching, respectively.

The "bottom-contact" (bottom source and drain contact, bottom gate contact) type OFET devices (Fig. 1) were fabricated as follows. Heavily doped $\mathrm{n}^{+}$-Si $(100)$ wafers with a thermally grown insulating $\mathrm{SiO}_{2}$ layer $(210$ $\mathrm{nm}$ thick) were used as the substrates. Gold drain and source electrodes $(50 \mathrm{~nm}$ thick) were patterned on the $\mathrm{Si} / \mathrm{SiO}_{2}$ substrate using a photolithography technique. For a typical device, the drain-source channel length $(L)$ and width $(W)$ were $10 \mu \mathrm{m}$ and $2.0 \mathrm{~cm}$, respectively. The stretched and unstretched $\mathrm{P} 3 \mathrm{HT} / \mathrm{PVA}$ films were applied by pressing to the device in the desired orientations.

Polarized UV-Vis absorption spectra were recorded on a Shimadzu UV-3100 spectrometer under ambient conditions. The dichroic ratio $\left(R_{\mathrm{d}}\right)$ was determined using the equation $R_{\mathrm{d}}=A_{/ / \max } / A_{\perp \max }$, where $A_{/ / \text {max }}$ and $A_{\perp \max }$ are absorbances at $\lambda_{\max }$ obtained for incident lights with electric vectors polarized parallel and orthogonal to the stretched direction of the films, respectively.

The electrical properties of the OFET devices were measured using an Agilent 4155C semiconductor parameter analyzer under ambient conditions. Field-effect mobility was calculated in the saturated regime $\left(V_{\mathrm{d}}=\right.$ $-100 \mathrm{~V})$ of the drain current. The current on/off ratio $\left(I_{\text {on }} / I_{\text {off }}\right)$ was determined from the maximum $\left(I_{\text {on }}\right)$ and minimum $\left(I_{\text {off }}\right)$ value of the $I_{\mathrm{d}}$.

\section{Results and Discussion}

Figure 2 shows polarized UV-Vis absorption spectra of the stretched P3HT/PVA film $\left(S_{\mathrm{r}}=1.9\right)$. $A_{/ /}$and $A_{\perp}$ represent the absorption spectra observed for the planepolarized light with electric vectors parallel and orthogonal to the stretching direction of the P3HT/PVA film, respectively. For unstretched film, $A_{/ /}$and $A_{\perp}$ are essentially the same, suggesting that $\mathrm{P} 3 \mathrm{HT}$ is randomly oriented in the unstretched film. After stretching the P3HT/PVA film, large dichroism was observed in the region from 400 to $700 \mathrm{~nm}$ with $R_{\mathrm{d}}=3.92$. Since the transition dipole moment of polythiophene is generally polarized along the polythiophene backbone, P3HT is consider to be oriented parallel to the stretching direction.

Figure 3 shows the relationship between $R_{\mathrm{d}}$ and $S_{\mathrm{r}}$ obtained with the stretched P3HT film on the supporting PVA film. The value of $R_{\mathrm{d}}$ increases with increasing the $S_{\mathrm{r}}$ value and reached 9.1 at $S_{\mathrm{r}}=3.7$. The supporting PVA

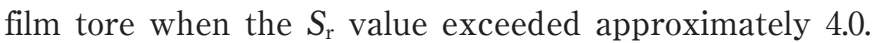
These results are consistent with the various $\pi$-conjugated polymers. ${ }^{9-11)}$

Bottom-contact type OFET devices were fabricated with unstretched and stretched P3HT/PVA films. Figure 4 represents typical plots of $I_{\mathrm{d}} v s$. $V_{\mathrm{d}}$ at various gate voltages for the OFET devices based on the stretched P3HT film $\left(S_{\mathrm{r}}=3.4\right)$ applied parallel to the electric field. The channel conductance increases as $V_{\mathrm{g}}$ becomes more negative, meaning that the P3HT film behaves as a $p$-type semiconductor. The field-effect

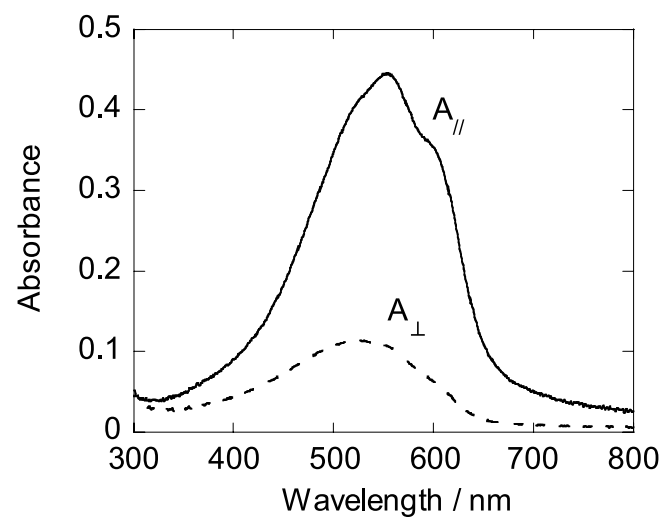

Fig. 2 Polarized UV-Vis absorption spectra of the stretched P3HT films $\left(S_{\mathrm{r}}=1.9\right)$ on the PVA substrates. $A_{/ /}$ (solid line) and $A_{\perp}$ (broken line) represent the spectra obtained for incident light with electric vectors polarized parallel and orthogonal to the stretching direction of the $\mathrm{P} 3 \mathrm{HT} / \mathrm{PVA}$ films, respectively.

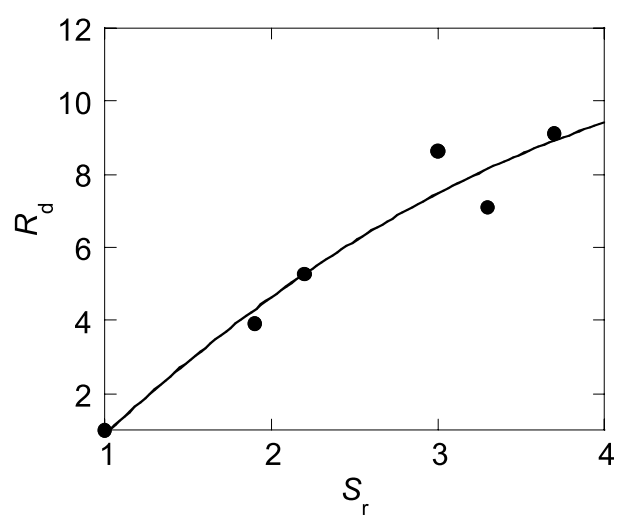

Fig. 3 Dependence of $R_{\mathrm{d}}$ on $S_{\mathrm{r}}$ for the stretched $\mathrm{P} 3 \mathrm{HT} / \mathrm{PVA}$ film.

mobility calculated using $I_{\mathrm{d}}$ in the saturation regions was $6 \times 10^{-2} \mathrm{~cm}^{2} \mathrm{~V}^{-1} \mathrm{~s}^{-1}$ with $I_{\text {on }} / I_{\text {off }}=\sim 10^{5}$ and $V_{\text {th }}=-13 \mathrm{~V}$. This value of mobility is several times higher than that of the orthogonal film, while the values of $V_{\text {th }}$ were almost the same.

Relationship between field-effect mobility and $S_{\mathrm{r}}$ obtained with the OFET devices with the unstretched $\left(S_{\mathrm{r}}=1.0\right)$ and stretched P3HT films applied orthogonally and parallel to the electric field is shown in Fig. 5. For the unstretched P3HT-based device, the field-effect mobility was about $1.3 \times 10^{-2} \mathrm{~cm}^{2} \mathrm{~V}^{-1} \mathrm{~s}^{-1}$. The mobility increased with increasing $S_{\mathrm{r}}$ value for the parallel devices but decreased for the orthogonal devices. This suggests that the intramolecular charge migration in the polymer backbone is faster than intermolecular charge migration. Interestingly, this charge migration direction is different from small molecules-based high performance OFETs. For small molecules, it is accepted that charge migration in the film with perpendicularly oriented molecules occurs via the overlapping of frontier molecular orbitals between the neighboring molecules. ${ }^{14)}$ Further investigations about the charge migration mechanism are underway. 


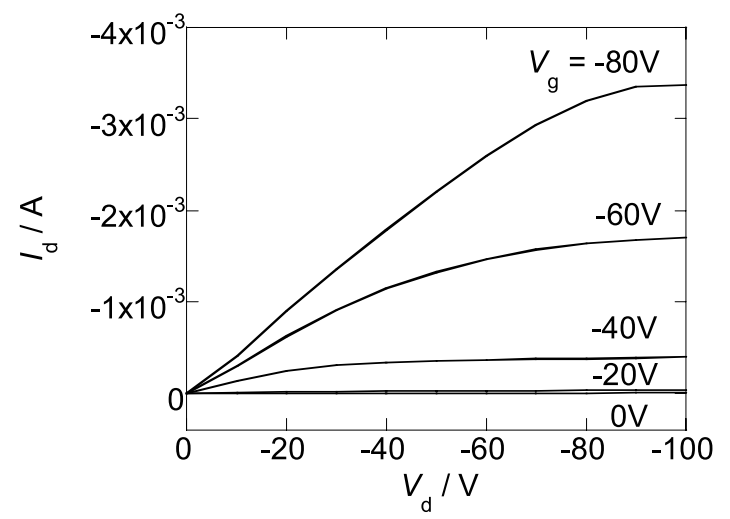

Fig. $4 I_{\mathrm{d}} v s$. $V_{\mathrm{d}}$ curves at different gate biases for the OFET devices with the stretched P3HT film $\left(S_{\mathrm{r}}=3.4\right)$ applied parallel to the electric field.

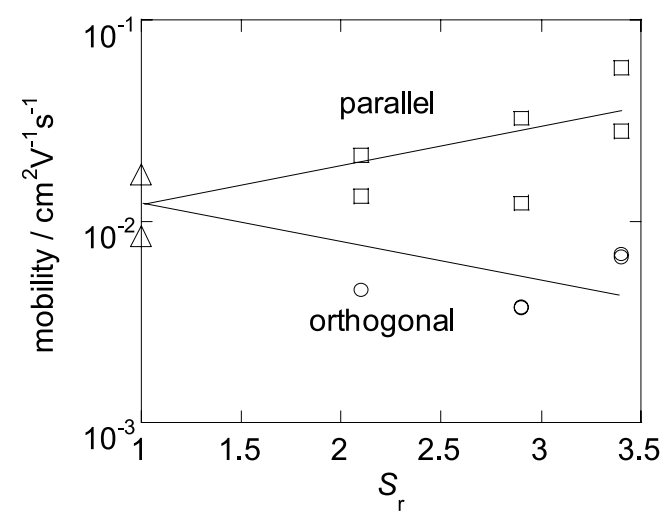

Fig. 5 Relationship between field-effect mobility and $S_{\mathrm{r}}$ obtained with the OFET devices with the unstretched $(\triangle)$ and stretched P3HT films applied $(\square)$ parallel and $(\bigcirc)$ orthogonal to the electric field.

\section{Conclusion}

In summary, we have successfully fabricated OFETs with mechanically stretched P3HT films acting as the active layers. After stretching the P3HT film, large dichroism was observed in the polarized UV-Vis absorption spectra. The field-effect mobility was highly anisotropic, with larger mobility along the stretched direction of the films. The mobility increased with the stretch ratio $\left(S_{\mathrm{r}}\right)$ for the parallel devices, while it decreased as the $S_{\mathrm{r}}$ value increased for the orthogonal devices. The mechanical stretching method is a promising technique for the processing of uniaxially oriented $\pi$ conjugated polymer films in order to fabricate high performance OFETs.

\section{Acknowledgment}

This work is partly supported by a Grant-in-Aid for Scientific Research from the Japan Society for the Promotion of Science (19550181).

\section{References}

1) Y. Noguchi, T. Sekitani, T. Yokota, and T. Someya, Appl. Phys. Lett., 93, 043303 (2008).

2) S. H. Ko, H. Pan, C. P. Grigoropoulos, C. K. Luscombe, J. M. J. Frechet, and D. Poulikakos, Appl. Phys. Lett., 90, 141103 (2007).

3) S. Allard, M. Forster, B. Souharce, H. Thiem, and U. Scherf, Angew. Chem. Int. Ed., 47, 4070 (2008).

4) S. Handa, E. Miyazaki, K. Takimiya, and Y. Kunugi, J. Am. Chem. Soc., 129, 11684 (2007).

5) T. Izawa, E. Miyazaki, and K. Takimiya, Adv. Mater., 20, 3388 (2008).

6) S.-Z. Weng, W.-S. Hu, C.-H. Kuo, Y.-T. Tao, L.-J. Fan, and Y.-W. Yang, Appl. Phys. Lett., 89, 172103 (2006).

7) S.-J. Kang, Y.-Y. Noh, K.-J. Baeg, J. Ghim, J. S. Kim, J. H. Park, and K. Cho, Appl. Phys. Lett., 92, 052107 (2008).

8) W.-Y. Chou and H.-L. Cheng, Adv. Funct. Mater., 14, 811 (2004).

9) H. Horiuchi, T. Ishihara, S. Tsunoda, D. Morishita, T. Takei, T. Okutsu, T. Seko, H. Sugino, Y. Kawakami, H. Watanabe, M. Abe, S. Takegami, and H. Hiratsuka, J. Phys. Chem. B, 110, 9072 (2006).

10) T. Yamamoto, T. Maruyama, and K. Kubota, Chem. Lett., 18, 1951 (1989).

11) M. Onoda, Y. Manda, and K. Yoshino, Jpn. J. Appl. Phys., 29, 1490 (1990).

12) H. Sirringhaus, P. J. Brown, R. H. Friend, M. M. Nielsen, K. Bechgaard, B. M. W. Langeveld-Voss, A. J. H. Spiering, R. A. J. Janssen, E. W. Meijer, P. Herwig, and D. M. de Leeuw, Nature, 401, 685 (1999).

13) M. Surin, Ph. Leclère, R. Lazzaroni, J. D. Yuen, G. Wang, D. Moses, A. J. Heeger, S. Cho, and K. Lee, J. Appl. Phys., 100, 033712 (2006).

14) K. Takimiya, Y. Kunugi, and T. Otsubo, Chem. Lett., 36, 578 (2007). 